\title{
A New Method of Fault Feature Extraction Based on Hierarchical Dispersion Entropy
}

\author{
Peng Chen, ${ }^{1,2}$ Xiaoqiang Zhao $\mathbb{D D}^{1,3,4}$ and HongMei Jiang ${ }^{1,3,4}$ \\ ${ }^{1}$ College of Electrical and Information Engineering, Lanzhou University of Technology, Lanzhou 730050, China \\ ${ }^{2}$ College of Electrical and Electronic Engineering, Lanzhou Petrochemical Polytechnic, Lanzhou 730060, China \\ ${ }^{3}$ Key Laboratory of Gansu Advanced Control for Industrial Processes, Lanzhou University of Technology, Lanzhou 730050, China \\ ${ }^{4}$ National Experimental Teaching Center of Electrical and Control Engineering, Lanzhou University of Technology, \\ Lanzhou 730050, China
}

Correspondence should be addressed to Xiaoqiang Zhao; xqzhao@lut.cn

Received 23 September 2020; Revised 5 January 2021; Accepted 18 January 2021; Published 26 February 2021

Academic Editor: Lei Su

Copyright (c) 2021 Peng Chen et al. This is an open access article distributed under the Creative Commons Attribution License, which permits unrestricted use, distribution, and reproduction in any medium, provided the original work is properly cited.

In the process of fault feature extraction of rolling bearing, the feature information is difficult to be extracted fully. A novel method of fault feature extraction called hierarchical dispersion entropy is proposed in this paper. In this method, the vibration signals firstly are decomposed hierarchically. Secondly, dispersion entropies of different nodes are calculated. Hierarchical dispersion entropy can realize the comprehensive feature extraction of the high- and low-frequency band information of vibration signals and overcome the problems that dispersion entropy and multiscale dispersion entropy are insufficient to extract the fault feature information of vibration signals. The feasibility of hierarchical dispersion entropy is obtained by analyzing the hierarchical dispersion entropy of Gaussian white noise and compared with the multiscale dispersion entropy of Gaussian white noise. Meanwhile, a fault diagnosis method for rolling bearings based on hierarchical dispersion entropy and $k$-nearest neighbor (KNN) classifier is developed. Finally, the superiority of the proposed fault diagnosis method is verified in the realization of fault diagnosis of the rolling bearing in different positions and different degrees of damage.

\section{Introduction}

As a key component of rotating machinery, the failure rate of rolling bearing accounts for $30 \%-40 \%$ of rotating machinery failure [1]. So it is very important to monitor and diagnose the fault of rolling bearing to ensure the safe and reliable operation of the rotating machinery. However, the rolling bearing often runs in different working conditions and worse environments, which makes the vibration signals have nonlinear and nonstationary characteristics. How to effectively extract fault features from the vibration signals is very crucial [2].

In recent years, many nonlinear dynamic analysis methods, such as approximate entropy [3], sample entropy [4], fuzzy entropy [5], and permutation entropy [6] are widely used in fault feature extraction due to their abilities to measure the complexity of time series. Zheng et al. [7] firstly realized the decomposition of vibration signals through local characteristicscale decomposition (LCD). Then, the fault features of the modal components through fuzzy entropy are extracted to realize fault diagnosis of rolling bearing. Zhong et al. [8] extracted the fault features by sample entropy after empirical mode decomposition (EMD) of vibration signals to realize fault diagnosis of rolling bearing. Aiming at the parameter selection of multiscale permutation entropy, Wang et al. [9] proposed a method of optimizing parameters of multiscale permutation entropy to extract fault features. Li et al. [10] improved multiscale permutation entropy to extract fault features which were inputted into binary tree support vector machine to realize fault diagnosis of rolling bearing. Above entropies and their improvement are widely used in fault feature extraction, but the relationship between vibration amplitudes is not considered. So Rostaghi and Azami [11] proposed the dispersion entropy to characterize the complexity of time series 
which was proved better than the permutation entropy and sample entropy in the dataset fields of biology and finance. Because the dispersion entropy is not only efficient in the calculation but also it can describe the relationship between vibration amplitudes. Based on the dispersion entropy which can describe the relationship between vibration amplitudes, Mostafa [12] applied the dispersion entropy to the fault diagnosis of rotating machinery, which showed the dispersion entropy was better than the permutation entropy and the approximation entropy such as more stable in the detection of high SNR signals. However, the dispersion entropy could not extract multiscale fault features of signals. For this, Yi et al. [13] proposed the multiscale dispersion entropy which could realize fault feature extraction of vibration signals under multiscale. Luo et al. [14] proposed refined composite multi-scale dispersion entropy to realize fault diagnosis of rolling bearing by improving coarsening process. This method realized the feature extraction in the multiscale of vibration signals. However, literature $[15,16]$ pointed out that multiscale entropy could only extract the feature information of vibration signals in the low-frequency band and ignore the feature information in the high-frequency band. But the fault information of vibration signals of rolling bearing not only exists in the low-frequency band but also in the high-frequency band.

Therefore, in order to achieve a comprehensive fault feature extraction of rolling bearing, a new fault feature extraction method named hierarchical dispersion entropy is proposed. The fault features of vibration signals of rolling bearing are extracted by the hierarchical dispersion entropy and inputted into the KNN classifier [17] to realize fault diagnosis. The main contributions of this paper are as follows:

(1) Compared with traditional methods, such as sample entropy, approximate entropy, and permutation entropy, dispersion entropy has the advantage of measuring the relationship between vibration signal amplitudes. In this paper, the hierarchical dispersion entropy is proposed by introducing hierarchical decompose based on dispersion entropy. Hierarchical dispersion entropy can extract feature information from the high-frequency band and low-frequency band. It overcomes the problem that multiscale dispersion entropy could not extract fault information from high-frequency band.

(2) The hierarchical dispersion entropy could extract more fault features. So it is applied in the fault feature extraction of rolling bearing vibrations. The method of fault diagnosis of hierarchical dispersion entropy combined with KNN is realized.

This paper is organized as follows: Section 2 presents dispersion entropy and multiscale dispersion entropy. Section 3 is the theory of hierarchical dispersion entropy and its parameter selection and simulation analysis. Section 4 is the fault diagnosis of rolling bearing based on hierarchical dispersion entropy. Section 5 is experimental verification. Section 6 summarizes the conclusions.

\section{The Theory of Dispersion Entropy}

2.1. Dispersion Entropy. Dispersion entropy is a method which can characterize the irregularity of time series [11]. The greater the value of dispersion entropy, the higher the irregularity of time series. On the contrary, the smaller the value of dispersion entropy, the lower the irregularity of time series. The calculation steps of dispersion entropy for the time series $\mathbf{x}=\left\{x_{i}, i=1,2, \ldots, N\right\}$ are as follows:

(1) The normal distribution function (1) is selected to map the time series $\mathbf{x}$ to $\mathbf{y}=\left\{y_{j}, j=1,2, \ldots, N\right\}$ and $y_{j} \in(0,1)$.

$$
y_{j}=\frac{1}{\sigma \sqrt{2 \pi}} \int_{-\infty}^{x_{j}} e^{\left(\left(-(t-\mu)^{2}\right) / 2 \sigma^{2}\right) \mathrm{d} t}
$$

where $u$ and $\sigma$, respectively, represent mean and standard deviations of time series $x$.

(2) $y_{j}$ is mapped into integer indices between $[1,2, \ldots, c]$ by linear expression.

$$
\mathbf{z}_{j}^{c}=\operatorname{round}\left(c \cdot y_{j}+0.5\right)
$$

where round is the rounding function and cis the number of classes after mapping.

(3) The embedded vector $z_{i}^{m, c}$ is calculated by the following equation:

$$
\mathbf{z}_{i}^{m, c}=\left\{z_{i}^{c}, z_{i+d}^{c}, \ldots, z_{i+(m-1) d}^{c}\right\},
$$

where $i=1,2, \ldots, N-(m-1) d, m$ represents the embedded dimension, and drepresents the delay time.

(4) The dispersion entropy patterns $\pi_{v_{0}, v_{1}, \ldots, v_{m-1}}(v=$ $1,2, \ldots, c)$ of each time series $z_{i}^{m, c}$ are calculated. $c^{m}$ is the number of possible patterns, $z_{i}^{c}=v_{0}, z_{i+d}^{c}=$ $v_{1}, \ldots, z_{i+(m-1)}^{c} d=v_{m-1}$.

(5) For ${ }^{m}$ dispersion patterns, the probability of each dispersion pattern $\pi_{v_{0} v_{1} \ldots v_{m-1}}$ is as follows:

$$
p\left(\pi_{v_{0} v_{1} \ldots v_{m-1}}\right)=\frac{\text { number }\left(\pi_{v_{0} v_{1} \ldots v_{m-1}}\right)}{N-(m-1) d},
$$

where number $\left(\pi_{v_{0} v_{1} \ldots v_{m-1}}\right)$ is the number of dispersion patterns $\pi_{v_{0} v_{1} \ldots v_{m-1}} \cdot p\left(\pi_{v_{0} v_{1} \ldots v_{m-1}}\right)$ equals to the number of dispersion patterns $\pi_{v_{0} v_{1} \ldots v_{m-1}}$ divided by the number of elements in $z_{i}^{m, c}$.

(6) Dispersion entropy of time series is defined as follows: 


$$
D E(x, m, c, d)=-\sum_{\pi=1}^{c^{m}} p\left(\pi_{v_{0} v_{1} \ldots v_{m-1}}\right) \ln \left(p\left(\pi_{v_{0} v_{1} \ldots v_{m-1}}\right)\right) .
$$

2.2. Multiscale Dispersion Entropy. In 2002, Costa et al. [18] proposed a multiscale entropy method which characterized the complexity of time series under multiscale conditions. Azami et al. [19] introduced the multiscale analysis method and proposed the multiscale dispersion entropy. The calculation steps of multiscale dispersion entropy are as follows:

(1) $y^{\tau}(j)$ is obtained by coarsening original sequence $x(i)$ as follows:

$$
y^{\tau}(j)=\frac{1}{\tau} \sum_{i=(j-1) \tau+1}^{j \tau} x(i), \quad 1<j<\frac{\tau}{N}
$$

where $\tau$ is the scale factor, $\tau=1,2, \ldots, n$.

(2) According to the calculation process of dispersion entropy in Section 2.1, the multiscale dispersion entropy is obtained by analyzing the dispersion entropy of coarse-grained time series $y^{\mathcal{\tau}}(j)$.

Thus, it may be known that the key step of calculation of multiscale dispersion entropy is the coarse-grained of original time series. The coarsening process is to sum time series according to different scales and then average them to form a new time series. According to the analysis of literature $[15,16]$, we know that multiscale dispersion entropy only obtains the low-frequency information of time series, but ignores the high-frequency information. In fact, the fault information contained in vibration signals not only exists in the high-frequency band but also in the low-frequency band.

\section{The Theory of Hierarchical Dispersion Entropy}

3.1. Hierarchical Dispersion Entropy. Hierarchical dispersion entropy is proposed by introducing hierarchical analysis. The first step is to hierarchically decompose vibration signals. The second step is to get the dispersion entropy of different hierarchical nodes. The detailed calculation process is as follows:

Step 1. Vibration signals are decomposed hierarchically as follows:

(1) For the obtained vibration signals, the operator $Q_{j}$ is defined as follows:

$$
Q_{j}=\left[\begin{array}{ccccccc}
\frac{1}{2}\left(-\frac{1}{2}\right)^{j} & 0 & 0 & \ldots & 0 & 0 \\
0 & 0 & \frac{1}{2}\left(-\frac{1}{2}\right)^{j} & \ldots & 0 & 0 \\
0 & 0 & 0 & 0 & \ldots & \frac{1}{2}\left(-\frac{1}{2}\right)^{j}
\end{array}\right]_{2^{n-1} \times 2^{n}}
$$

where $j=1$ or 0 , the form of the matrix $Q_{j}$ is determined by the length of vibration signals. Therefore, the following operators are defined as follows:

$$
\begin{array}{ll}
Q_{0(\mu)}=\frac{\mu(2 j)+\mu(2 j+1)}{2}, & j=0,1,2, \ldots, 2^{n}, \\
Q_{1(\mu)}=\frac{\mu(2 j)-\mu(2 j+1)}{2}, & j=0,1,2, \ldots, 2^{n},
\end{array}
$$

$Q_{0}$ and $Q_{1}$ represent the low-frequency and highfrequency parts of the first layer of vibration signal decomposition. According to the operator $Q_{0}$ and $Q_{1}$, the reconstructed vibration signal is as follows:

$$
\boldsymbol{\mu}=\left\{Q_{0}(\boldsymbol{\mu})_{j}+Q_{1}(\boldsymbol{\mu})_{j}, Q_{0}(\boldsymbol{\mu})_{j}-Q_{1}(\boldsymbol{\mu})_{j}\right\},
$$

where $j=0,1,2, \ldots, 2^{n-1}$.

(2) $N$-dimensional $\quad$ vector $\left[\gamma_{1}, \gamma_{2}, \ldots \gamma_{n}\right] \in\{0,1\}$ is reconstructed. The integerecan be expressed by $\gamma_{j}$ as follows:

$$
e=\sum_{j=1}^{k} \gamma_{j} 2^{k-j}
$$

(3) According to the vector $\left[\gamma_{1}, \gamma_{2}, \ldots, \gamma_{n}\right]$, the nodes of each layer decomposition of vibration signal $u_{i}$ are defined as follows, and the hierarchical structure is shown in Figure 1.

Step 2. The calculation function of hierarchical dispersion entropy of different nodes is as follows:

$$
\operatorname{HDE}(k)=D E(\operatorname{data}, k, m, c, d),
$$

where data are the original vibration signals, $k$ is the number of decomposition order, mis the embedded dimension, cis the number of mapping class, and $d$ is the time delay.

It can be seen from the definition of operators that $Q_{0}$ and $Q_{1}$ represent the low frequency and the high frequency, which 


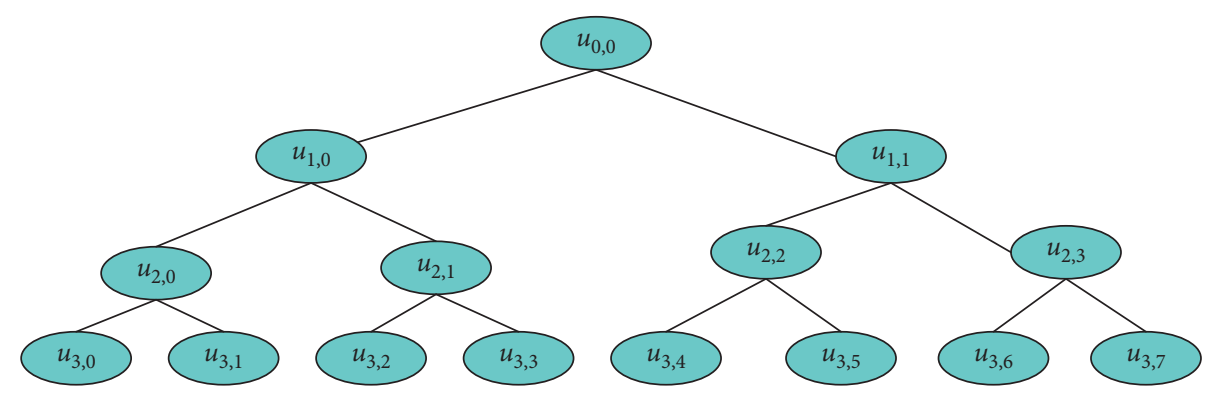

Figure 1: Time series decomposition diagram $(k=3)$.

are equivalent to the low pass and the high pass in wavelet analysis. The dispersion entropies of $u_{(1,0)}, u_{(2,0)}$, and $u_{(3,0)}$ on the left of Figure 1 are equivalent to the dispersion entropies of different scales in multiscale dispersion entropy. In essence, the dispersion entropy of $u_{k, 0}$ is the dispersion entropy of different scales in multiscale, which represents the low-frequency part of time series. The high-frequency part is on the right of Figure 1, which is ignored by the multiscale entropy. It can be seen that hierarchical dispersion entropy can not only extract the lowfrequency information of vibration signals but also extract the high-frequency information. Therefore, the ability of hierarchical dispersion entropy is better than multiscale dispersion entropy for extracting fault features of rolling bearing from the theoretical analysis.

3.2. Parameter Selection. In the process of calculating hierarchical dispersion entropy, the related parameters need to be set in advance. These parameters are decomposition order $k$, embedding dimension $m$, mapping classes $c$, and time delay $d$. When the $k$ value is small, the frequency band of vibration signals would be larger and the detailed fault feature information from low frequency to high frequency could not be obtained. If the $k$ value is large, the calculation efficiency of the algorithm would be reduced and the points involved in the calculation would be reduced. In general, the value of decomposition $k=3$ is selected [20].

At the same time, according to the influence of the parameters of the dispersion entropy in literature [12], the embedded dimension $m=2$, mapping classes $c=5$, and time delay $d=1$ are selected.

3.3. Simulation Analysis. The irregularity of Gaussian white noise in different frequency bands is basically unchanged [21]. Therefore, the feasibility of hierarchical dispersion entropy is verified by analyzing the hierarchical dispersion entropy of Gaussian white noise signal and compared with multiscale dispersion entropy of Gaussian white noise signal. The Gaussian white noise signal with a length of $N=2048$ is generated by simulation as shown in Figure 2. The sample frequency of the Gaussian white noise is $12 \mathrm{kHZ}$. The spectrum diagram can be obtained by Fourier transforming as shown in Figure 3.

The parameter settings $k=3$ and $\tau=8$ are used to extract the hierarchical dispersion entropy and multiscale dispersion entropy. At each scale, two kinds of entropy values are calculated for 100 groups of white noise generated randomly.

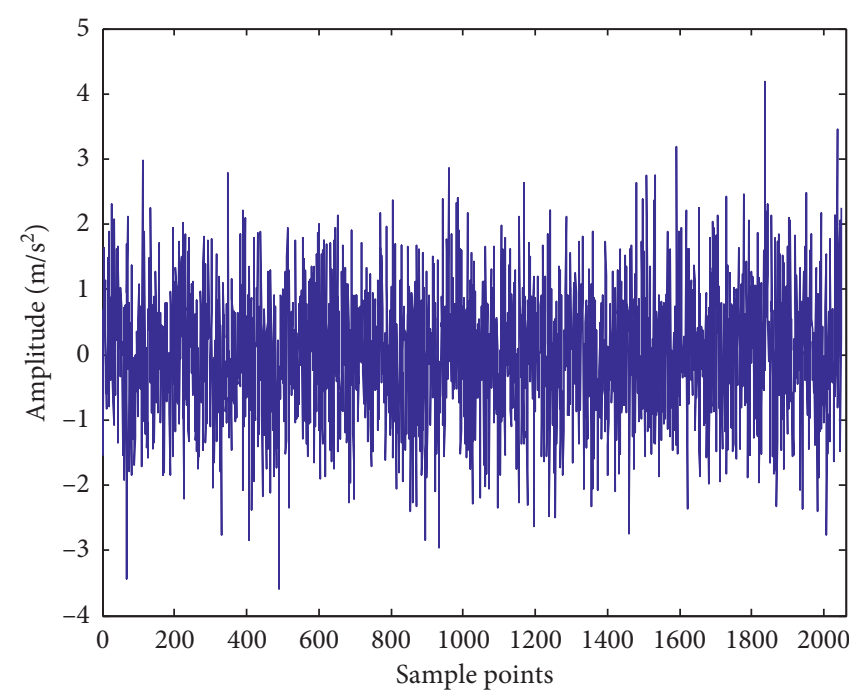

FIgURe 2: Gaussian white noise.

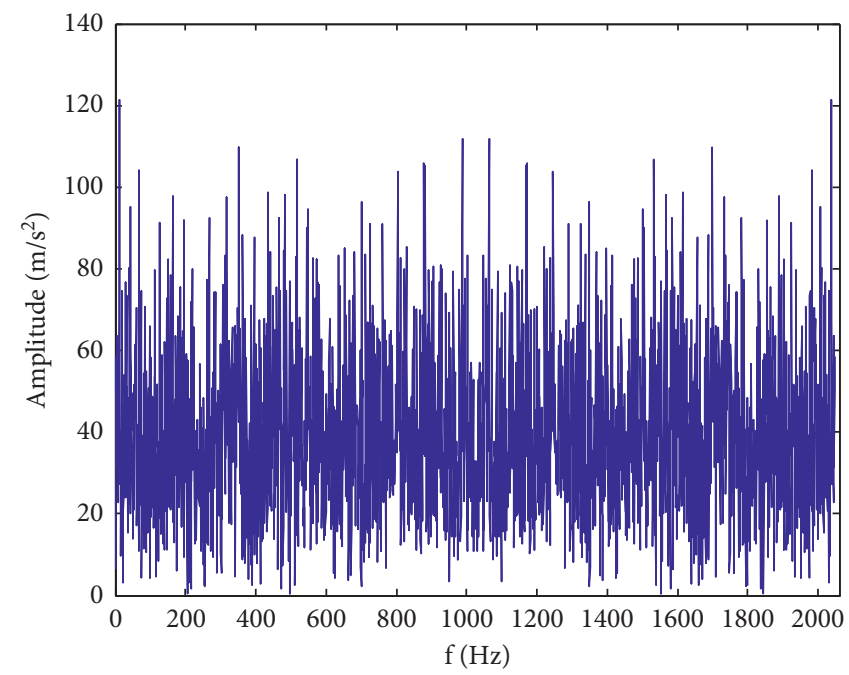

FIgURE 3: Spectrum of Gaussian white noise.

The error bars are shown in Figure 4. From Figure 4, we can see that the hierarchical dispersion entropy at different hierarchical nodes is basically unchanged. It is consistent with the fact that the complexity of Gaussian white noise in different frequency bands is basically unchanged. However, the multiscale dispersion entropy decreases with the scale 


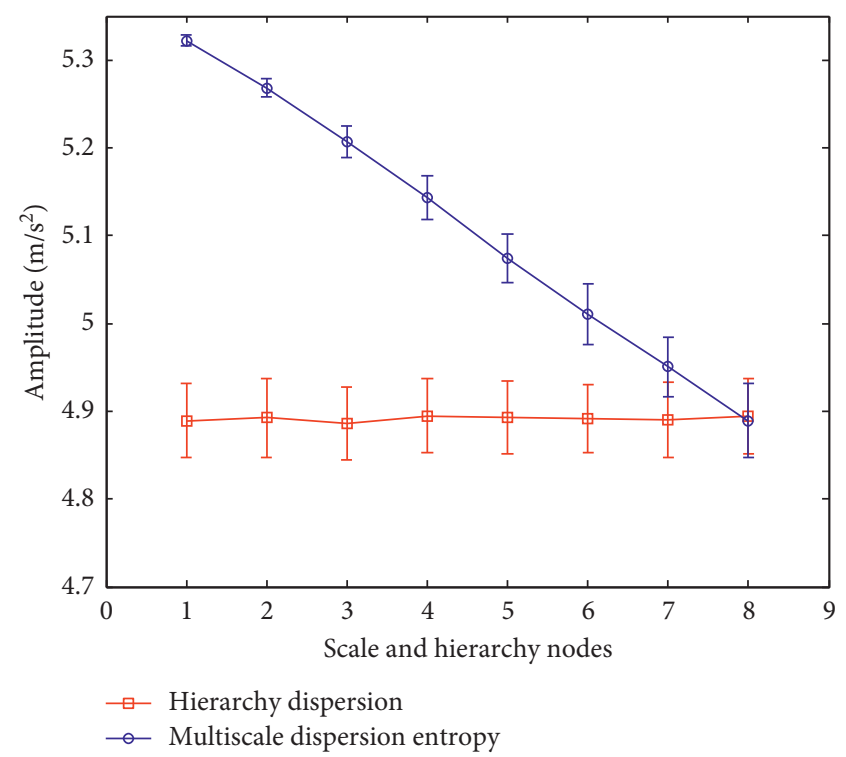

FIgURE 4: The error bars of hierarchical dispersion entropy and multiscale dispersion entropy.

increase which is not consistent with the fact that the complexity of Gaussian white noise in different frequency bands is basically unchanged. At the same time, the error bar of multiscale dispersion entropy is small when the scale is small. With the increase of scale, the error bar of multiscale dispersion entropy is larger. But the error bar of hierarchical dispersion entropy is basically unchanged. It means that the stability of multiscale dispersion entropy is poor and the stability of hierarchical dispersion entropy is better. To sum up, the hierarchical dispersion entropy could represent the complexity of signals and its performance is better than multiscale dispersion entropy.

\section{Fault Diagnosis of Rolling Bearing Based on Hierarchical Dispersion Entropy}

The complexity of vibration signal is different in a different position and damage degree of rolling bearing. Therefore, different fault features of vibration signals of rolling bearing can be extracted by the proposed hierarchical dispersion entropy. The fault diagnosis of rolling bearing is realized by inputting hierarchical dispersion entropies of vibration signals into the KNN classifier. The implementation steps of fault diagnosis are shown in Figure 5.

(1) Vibration signals of rolling bearing are collected.

(2) The hierarchical dispersion entropies of vibration signals in different states of rolling bearing are extracted. Eight different nodes are obtained by setting $k=3$. Other parameters are set $m=2, c=5$, and $d=1$.

(3) Fault features are divided into training samples and test samples.

(4) The training samples are inputted into the $\mathrm{KNN}$ classifier for model training.

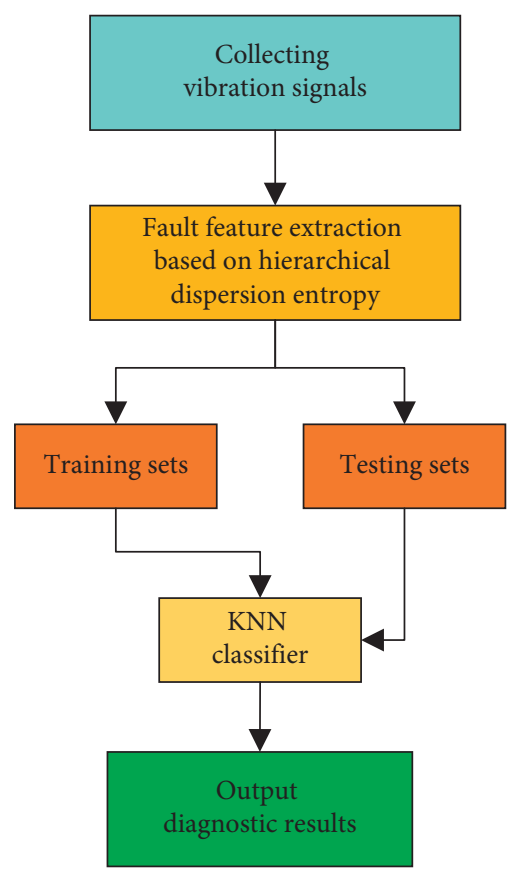

FIGURE 5: Flowchart of the hierarchical dispersion entropy + KNN method.

(5) The testing samples are inputted into the KNN classifier to get fault diagnosis results.

\section{Experimental Verification}

The rolling bearing experimental data of the Case Western Reserve University of the United States are selected [21]. The test system is shown in Figure 6 and the bearing is 62052RSJEMSKF deep groove ball bearing. Different fault settings of bearing inner ring, outer ring, and rolling element are made by electrodischarge machining (EDM) technology. The information of fault data is shown in Table 1.

5.1. Fault Diagnosis of Different Positions. At the sampling frequency of $12 \mathrm{kHz}$, the vibration signals of rolling bearing under normal (NR), inner ring fault (IRF), outer ring fault (ORF), and rolling element fault (BF) are collected when the load is $1.5 \mathrm{~kW}$ and the speed is $1750 \mathrm{r} / \mathrm{min}$. The time-domain waveform of vibration signals is shown in Figure 7.

We can see from Figure 7 that, it is difficult to directly identify different fault types of rolling bearing only through time-domain waveform. Firstly, the multiscale dispersion entropy of 120 samples (30 samples for each state) are extracted, and the mean value of the multiscale dispersion entropy is shown in Figure 8, where the scale $\tau=8$ is set and other parameters remain unchanged.

It can be seen from Figure 8 that multiscale dispersion entropy of vibration signals under normal conditions of rolling bearing is larger than that under fault conditions. This is due to the periodic impact of vibration signals in fault states is more regular than the randomness of vibration signals in the normal state. The multiscale dispersion 


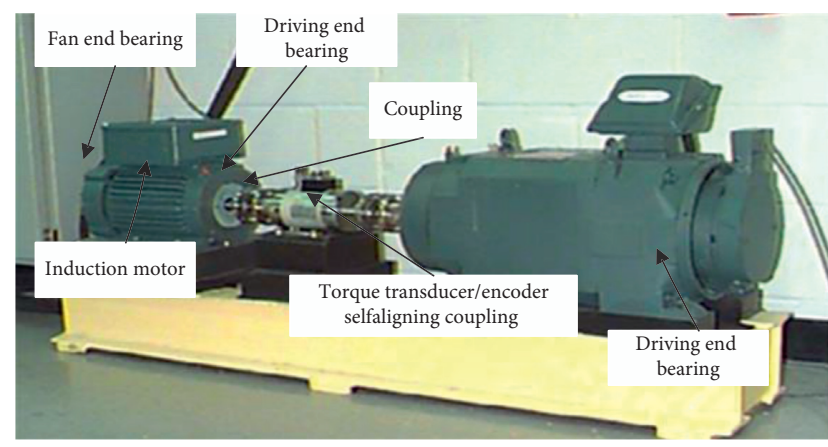

Figure 6: The test system for roll bearing.

TABLE 1: Fault data information.

\begin{tabular}{lcc}
\hline Fault type & Label & Fault diameter \\
\hline NR & 1 & 0 \\
\hline \multirow{3}{*}{ IRF } & 2 & $0.1778 \mathrm{~mm}$ \\
& 3 & $0.3556 \mathrm{~mm}$ \\
& 4 & $0.5334 \mathrm{~mm}$ \\
ORF & 5 & $0.7112 \mathrm{~mm}$ \\
& 6 & $0.1778 \mathrm{~mm}$ \\
& 7 & $0.3556 \mathrm{~mm}$ \\
& 8 & $0.5334 \mathrm{~mm}$ \\
BF & 9 & $0.1778 \mathrm{~mm}$ \\
& 10 & $0.3556 \mathrm{~mm}$ \\
& 11 & $0.5334 \mathrm{~mm}$ \\
\end{tabular}
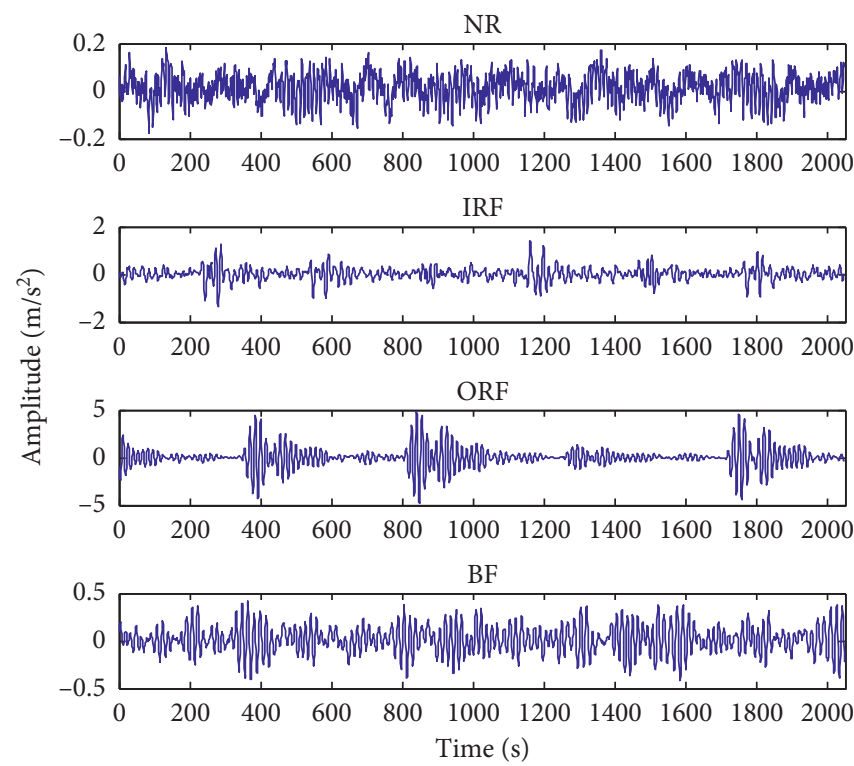

FIgURE 7: Time-domain waveform.

entropies of the balling and the inner ring are larger than that of the outer ring and are close to each other when the scale is small. Because the outer ring is fixed, but the balling and the inner ring run together with the bearing. This leads to balling and inner ring vulnerable to more noise interference in the path transmission and more irregularities.

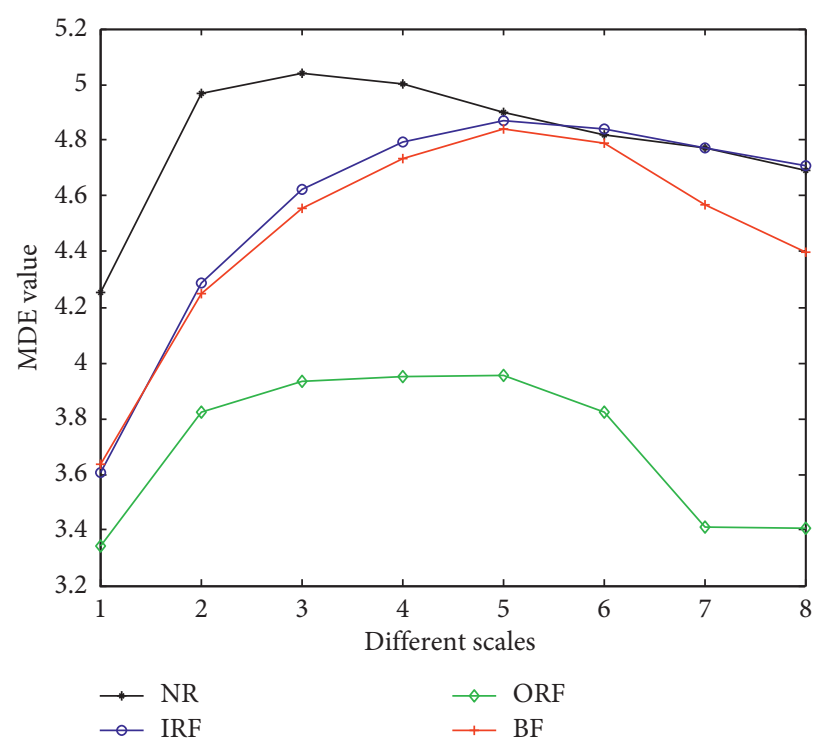

FIgURE 8: Multiscale dispersion entropy of different component samples.

The extracted multiscale dispersion entropy is inputted into the KNN classifier for fault diagnosis. We set the number of nearest neighbors $K=2$, where classification label 1 represents NR, classification label 2 represents IRF, classification label 3 represents ORF, and classification label 4 represents BF. The diagnosis results are shown in Figure 9.

From Figure 9, we can see that one sample of ball fault is considered as the inner ring fault. The overall diagnosis accuracy is $99.17 \%$.

The dispersion entropy is extracted from the same sample and is inputted into the KNN classifier for fault diagnosis as shown in Figure 10. Except for the scale $\tau$, the other parameters are the same as the multiscale dispersion entropy. The diagnosis accuracy is $75 \%$ and there are serious errors for the classification of inner ring and balling. Due to the rotation of inner ring and balling together, the fault impact signals interfere with each other. Dispersion entropy and multiscale dispersion entropy are not easy to extract the distinguishing features and are easy to cause misjudgment.

The fault features of rolling bearing are extracted by the proposed hierarchical dispersion entropy. The decomposition order is set $k=3$ and the other parameters are the same as the multiscale dispersion entropy and dispersion entropy. The hierarchical dispersion entropies of samples are shown in Figure 11.

Firstly, from Figure 11, we know that hierarchical dispersion entropy in normal state decreases with the increase of nodes in general. This indicates that the feature information mainly exists in the low frequency of vibration signals. Secondly, hierarchical dispersion entropy of vibration signals in node $=1$ (low frequency part) and node $=8$ (high-frequency part) is large in fault state. This indicates that the fault feature exists not only in the low frequency but also in the high frequency. Thirdly, the hierarchical dispersion entropy of the same node in different states is basically unchanged. This means that the stability of 


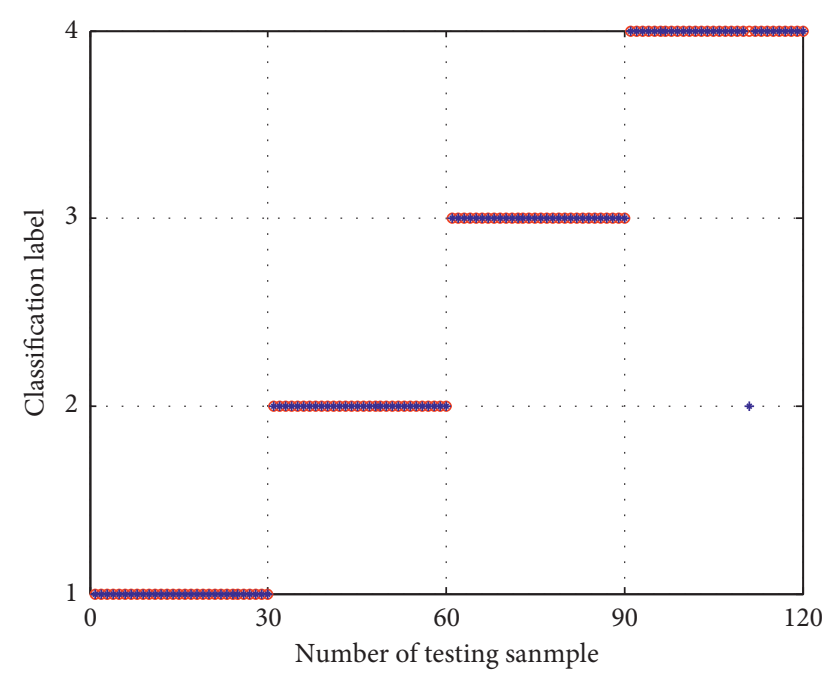

- Expect output

- Actual output

FIGURE 9: Diagnosis results of multiscale dispersion entropy.

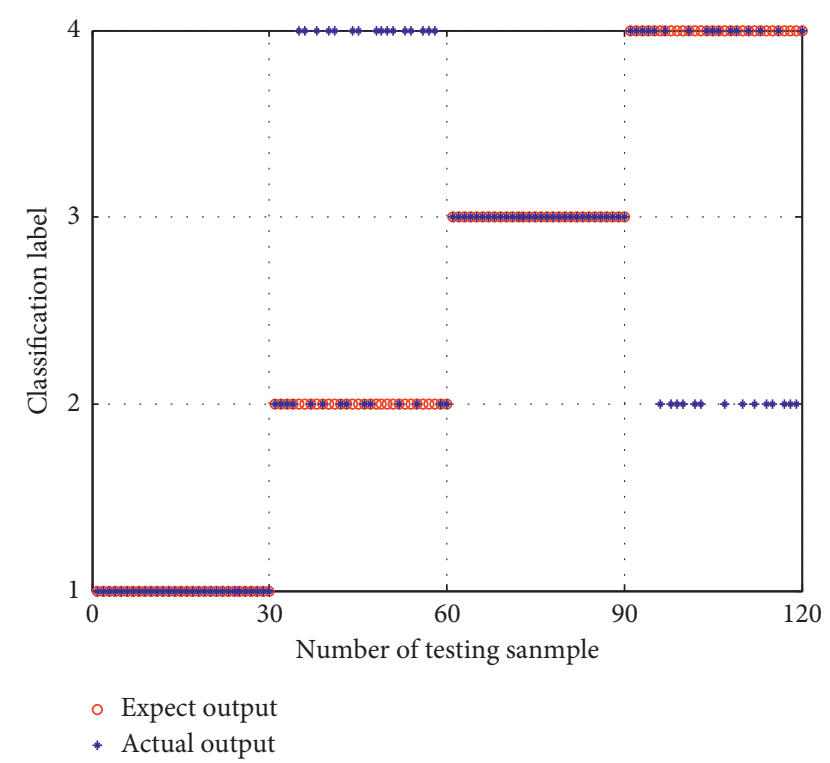

FIGURE 10: Diagnosis results of dispersion entropy.

hierarchical dispersion entropy is better, and the fault feature information exists in the high and low frequencies of vibration signals of rolling bearing. But multiscale dispersion entropy and dispersion entropy can only extract part of fault feature information. The hierarchical dispersion entropy can extract more comprehensive fault feature information which is more suitable for fault feature extraction of rolling bearing. Finally, the fault features extracted by hierarchical dispersion entropy are inputted into the KNN classifier for fault diagnosis under the same environment. The results are shown in Figure 12. We can see from Figure 12 that the accuracy of diagnosis result is $100 \%$ which is higher than $99.17 \%$ of multiscale dispersion entropy and $75 \%$ of dispersion entropy. The diagnostic accuracy of the three methods is shown

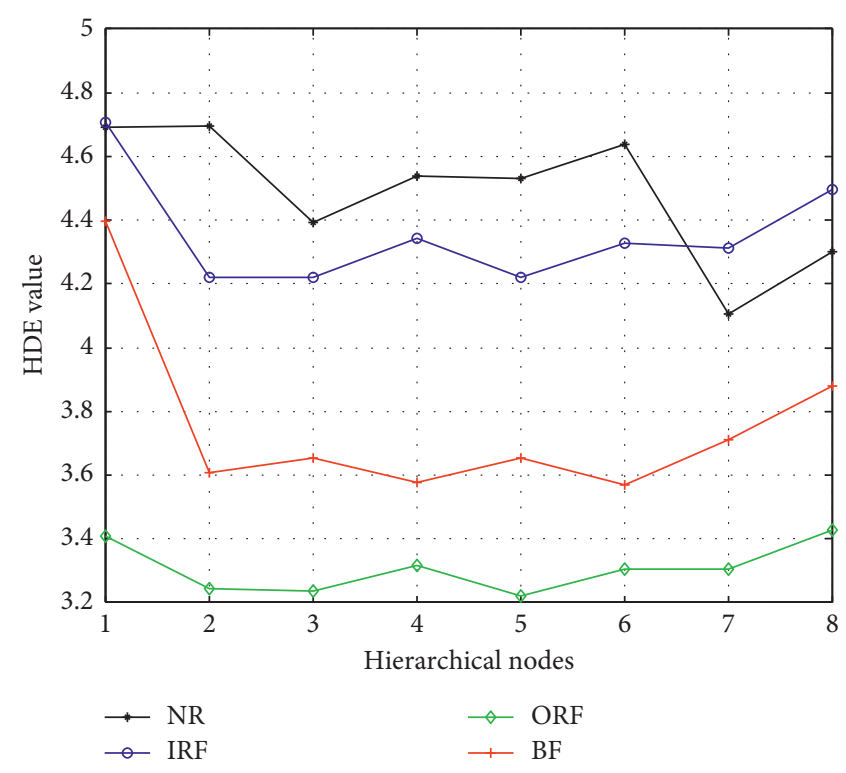

FIGURE 11: Hierarchical dispersion entropies of samples in four states.

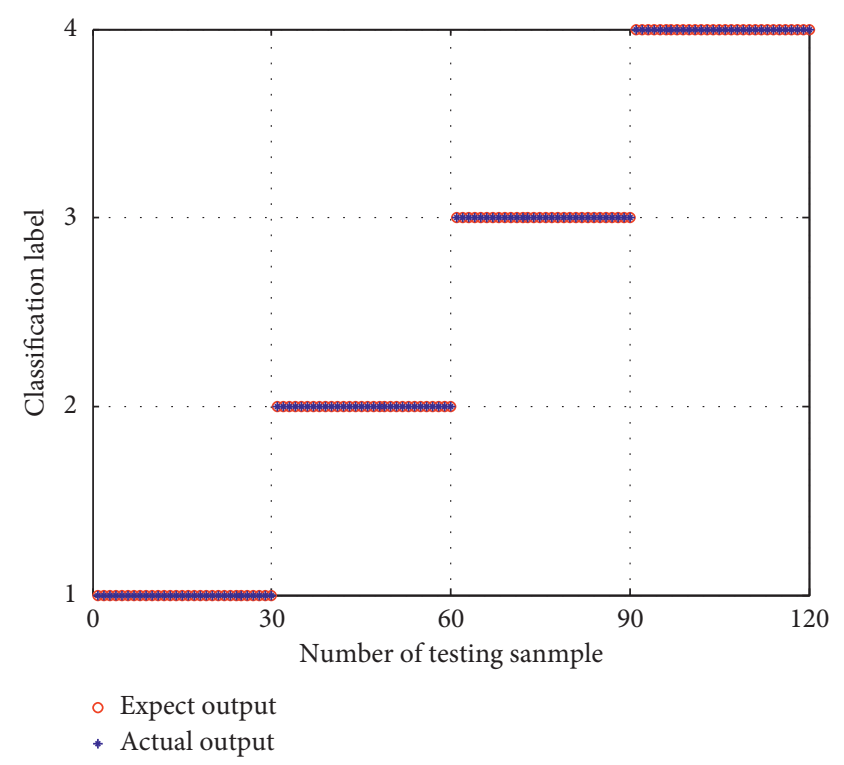

FiguRE 12: Diagnosis results of hierarchical dispersion entropy.

in Table 2. The effective feature extraction of inner ring and balling is realized. Therefore, hierarchical dispersion entropy is better than multiscale dispersion entropy and dispersion entropy for fault feature extraction.

5.2. Fault Diagnosis of Different Damage Degrees. In order to further verify the fault feature extraction ability of hierarchical dispersion entropy at the sampling frequency of 12 $\mathrm{kHZ}$, the vibration signals of different fault damage degrees of rolling bearing at the load of $0 \mathrm{~kW}$ and the speed of $1797 \mathrm{r} /$ min are shown in Figure 13. 12 different fault damage degrees and the corresponding classification labels are shown 
TABLE 2: The accuracy of fault diagnosis with different positions.

\begin{tabular}{lc}
\hline Method & Accuracy $(\%)$ \\
\hline Dispersion entropy + KNN & 75 \\
Multiscale dispersion entropy + KNN & 99.17 \\
Hierarchical dispersion entropy + KNN & 100
\end{tabular}
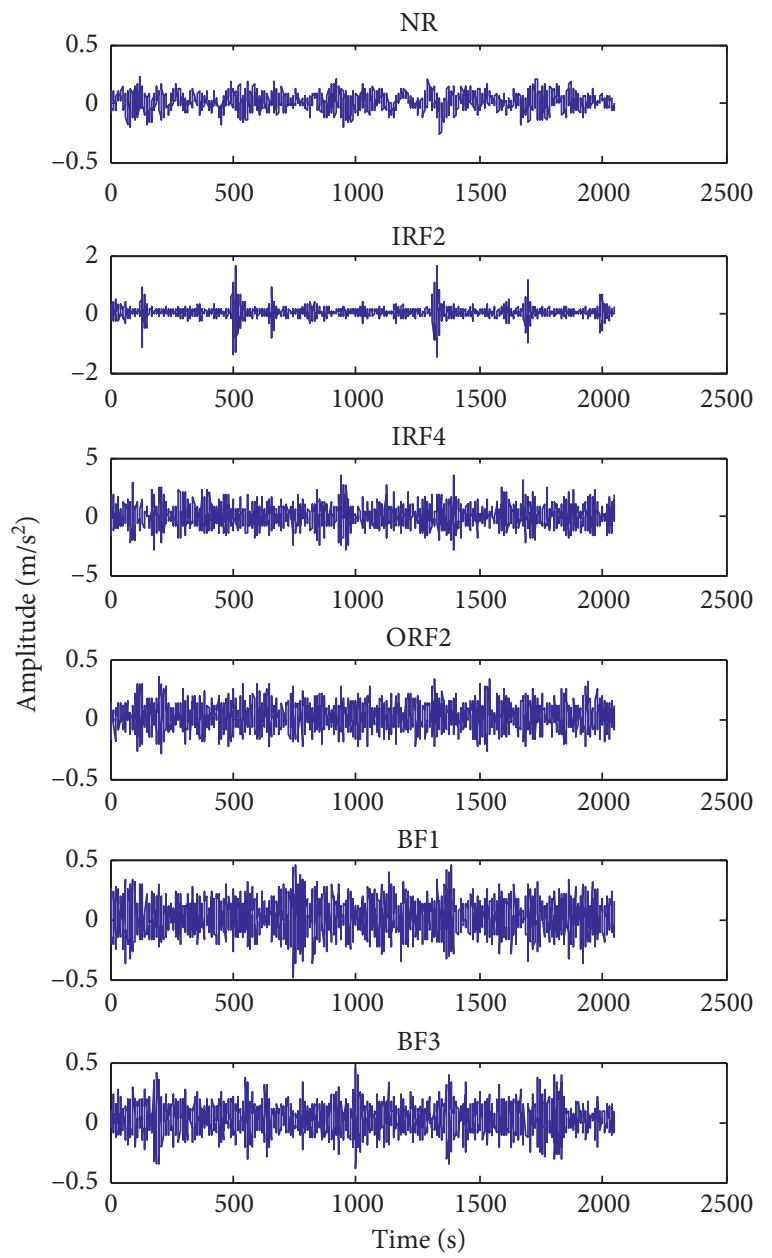

(a)
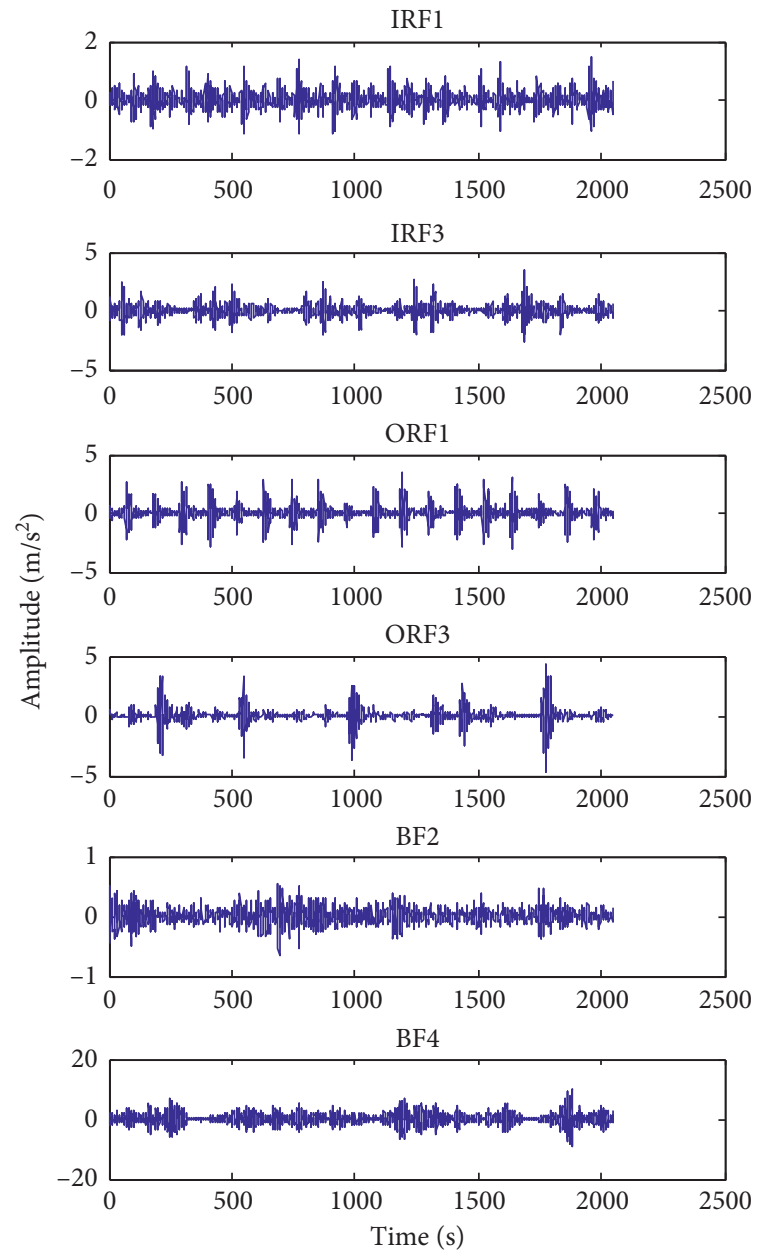

(b)

FIgURE 13: Time-domain waveforms of different damage degrees.

in Table 1. Among them, vibration signals of the outer ring 2, balling 1, and the balling 3 are greatly disturbed by noise and have no obvious impact.

Firstly, the fault features of different damage degrees are extracted by using dispersion entropy and multiscale dispersion entropy. Then, the fault features are inputted into the KNN classifier for fault diagnosis. The diagnosis results are shown in Figures 14 and 15, respectively. The parameters of dispersion entropy, multiscale dispersion entropy, and the KNN classifier are the same as fault feature extraction of different positions.

It can be seen from Figures 14 and 15 that fault diagnosis accuracies of different fault damage degrees by dispersion entropy and multiscale dispersion entropy are $63.33 \%$ and $73.67 \%$, respectively. Compared with the fault diagnosis of different positions, the fault diagnosis accuracies of different fault damage degrees by using dispersion entropy and multiscale dispersion entropy are greatly reduced. Because the fault signals with different damage degrees are more similar which make them more difficult to distinguish. At the same time, the two methods are less able to distinguish the signals with stronger similarity. Thus, it is not feasible to diagnose different damage faults directly by dispersion entropy and multiscale dispersion entropy. Similarly, the accuracy of fault diagnosis with different damage degrees by using hierarchical dispersion entropy is $98.33 \%$, and the results are shown in Figure 16. There is an error classification between the inner ring and the balling. The diagnosis accuracy of the three methods is shown in Table 3. Compared with the dispersion entropy and multiscale dispersion 


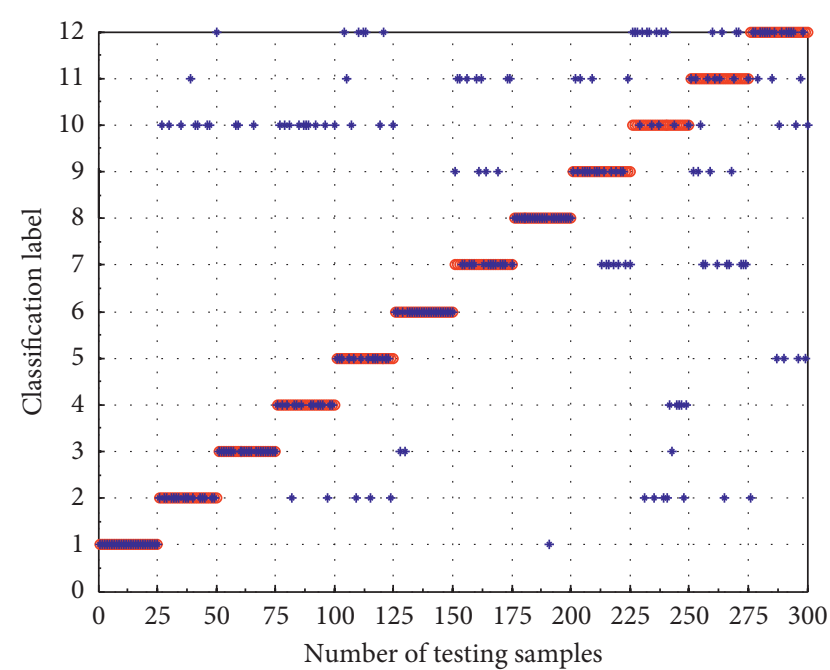

- Expect output

- Actual output

FIgURE 14: Diagnosis results of dispersion entropy.

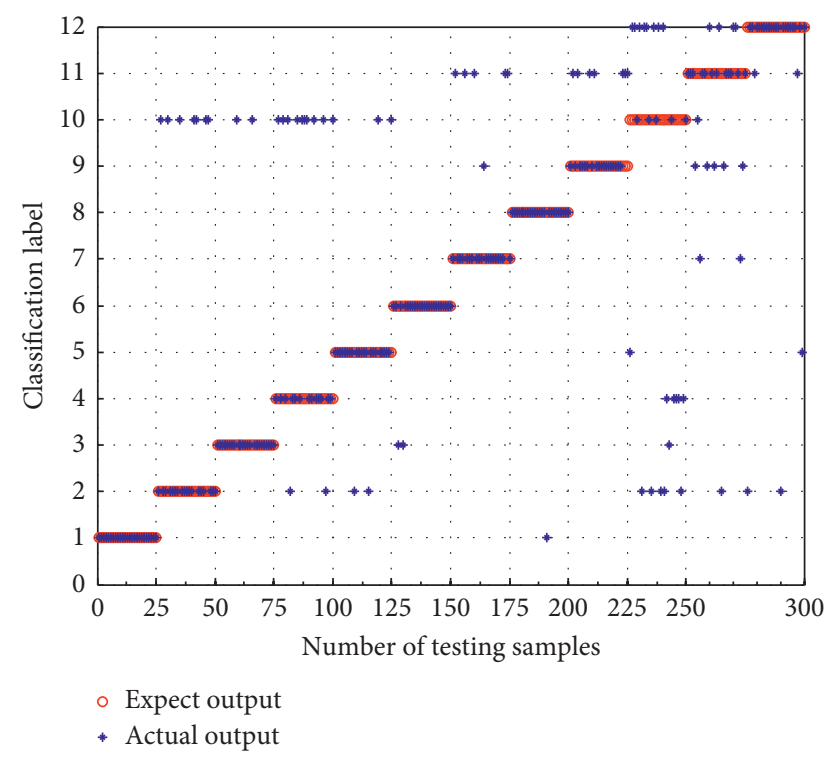

Figure 15: Diagnosis results of multiscale dispersion entropy.

entropy, the accuracy is greatly improved. This shows that hierarchical dispersion entropy can realize the diagnosis of different damage fault degrees and has a stronger ability to extract fault features. At the same time, the fault diagnosis accuracy of $100 \%$ can be achieved for the outer ring 2, the balling 1, and the Balling 3 with strong noise interference.

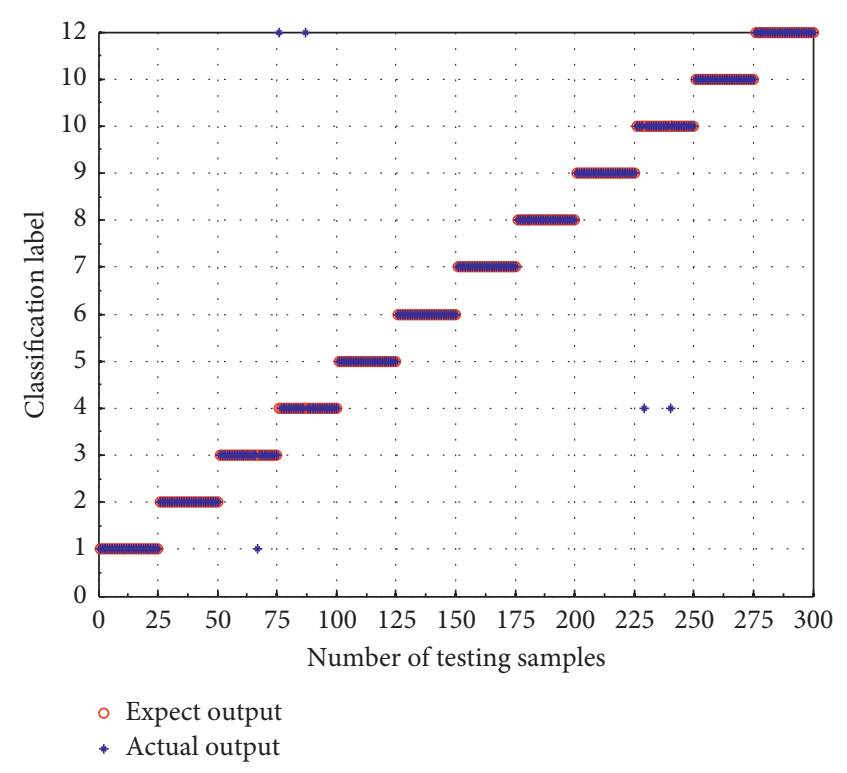

Figure 16: Diagnosis results of hierarchical dispersion entropy.

TABle 3: The accuracy of fault diagnosis with different damage degrees.

\begin{tabular}{lc}
\hline Method & Accuracy (\%) \\
\hline Dispersion entropy + KNN & 63.33 \\
Multiscale dispersion entropy + KNN & 73.67 \\
Hierarchical dispersion entropy + KNN & 98.33 \\
\hline
\end{tabular}

This further shows that hierarchical dispersion entropy has better fault feature extraction ability under strong noise.

To summarize, the fault diagnosis ability of dispersion entropy for different positions and damage degrees is poor. So it is not feasible to directly extract the fault features of vibration signals. The multiscale dispersion entropy has a high diagnostic accuracy of fault diagnosis for different positions, but its discrimination ability of different damage degrees is not satisfied. Hierarchical dispersion entropy not only achieves good results for fault diagnosis of different positions but also achieves satisfactory results for fault diagnosis of different damage degrees. Because it can extract the fault feature information of vibration signals in high- and low-frequency bands simultaneously. So hierarchical dispersion entropy has obvious advantages for fault features extraction of complex signals and is better than dispersion entropy and multiscale dispersion entropy. Finally, for the same dataset, the accuracies of the proposed method and other methods are shown in Table 4. It can be seen that the proposed method in this paper has a high-fault diagnosis rate and a good diagnostic accuracy in fault diagnosis of multiclassification. 
TABLE 4: Accuracy comparison of four methods.

\begin{tabular}{lcc}
\hline $\begin{array}{l}\text { The number of } \\
\text { fault type }\end{array}$ & Method & Accuracy (\%) \\
\hline 10 & PSO + DBN [22] & 97.8 \\
12 & IMDE + mRMR + ELM [23] & 95.15 \\
12 & RCMDE + mRMR + ELM [23] & 94.37 \\
12 & Hierarchical dispersion & 98.33 \\
\hline
\end{tabular}

\section{Conclusions}

In this paper, a rolling bearing fault diagnosis method based on hierarchical dispersion entropy and $\mathrm{KNN}$ is proposed. The main conclusions are as follows:

(1) Based on the dispersion entropy, this paper proposed the hierarchical dispersion entropy by combining with hierarchical decomposition.

(2) Hierarchical dispersion entropy can extract the highand low-frequency band characteristics of the signal. It solves the problem that dispersion entropy and hierarchical dispersion entropy cannot extract highfrequency band information of signal.

(3) Finally, the method combined with KNN can realize the fault diagnosis of rolling bearing in different positions and damage degrees. It provides a new idea for fault feature extraction of rolling bearing.

\section{Data Availability}

The datasets analysed during the current study are available in the https://csegroups.case.edu/bearingdatacenter/pages/ download-data-file link to datasets.

\section{Conflicts of Interest}

The authors declare that there are no conflicts of interest regarding the publication of this paper.

\section{Acknowledgments}

This work was supported by the National Natural Science Foundation of China (nos.61763029 and 61873116), the National Defense Basic Research Project of China (JCKY2018427C002), the Industrial Support and Guidance Project of Colleges and Universities of Gansu Province (no. 2019C-05), and the Open Fund Project of Key Laboratory of Gansu Advanced Control for Industrial Processes (no. 2019KFJJ01). The authors would like to thank Case Western Reserve University for providing motor bearing vibration data.

\section{References}

[1] P. Chen, X. Zhao, and Q. Zhu, "A novel classification method based on ICGOA-KELM for fault diagnosis of rolling bearing," Applied Intelligence, vol. 81, no. 50, pp. 2833-2847, 2020.

[2] Y. Lv, R. Yuan, and G. Song, "Multivariate empirical mode decomposition and its application to fault diagnosis of rolling bearing," Mechanical Systems and Signal Processing, vol. 81, no. 12, pp. 219-234, 2016.

[3] S. M. Pincus, "Approximate entropy as a measure of system complexity," Proceedings of the National Academy of Sciences, vol. 88, no. 6, pp. 2297-2301, 1991.

[4] J. S. Richman and J. R. Moorman, "Physiological time-series analysis using approximate entropy and sample entropy," American Journal of Physiology-Heart and Circulatory Physiology, vol. 278, no. 6, pp. 2039-2049, 2000.

[5] W. Chen and W. Zhuang, "Measuring complexity using FuzzyEn, ApEn, and SampEn," MedicEngineering \& Physics, vol. 89, no. 31, pp. 61-68, 2009.

[6] B. Christoph and P. Berndt, "Permutation entropy: a natural complexity measure for time series," MedicEngineering \& Physics, vol. 88, no. 17, pp. 102-174, 2002.

[7] J. Zheng, J. Cheng, and Y. Yang, "A rolling bearing fault diagnosis approach based on LCD and fuzzy entropy," Mechanism and Machine Theory, vol. 70, pp. 441-453, 2013.

[8] X. Y. Zhong, C. H. Zhao, H. J. Dong, X. M. Liu, and L. C. Zeng, "Rolling bearing fault diagnosis using sample entropy and 1.5 dimension spectrum based on EMD," Applied Mechanics and Materials, vol. 278-280, pp. 1027-1031, 2013.

[9] X. Wang, S. Si, Y. Wei, and Y. Li, "The optimized multi-scale permutation entropy and its application in compound fault diagnosis of rotating machinery," Entropy, vol. 21, no. 2, pp. 170-185, 2019.

[10] Y. Li, M. Xu, Y. Wei, and W. Huang, "A new rolling bearing fault diagnosis method based on multiscale permutation entropy and improved support vector machine based binary tree," Measurement, vol. 77, pp. 80-94, 2016.

[11] M. Rostaghi and H. Azami, "Dispersion entropy: a measure for time-series analysis," IEEE Signal Processing Letters, vol. 23, no. 5, pp. 610-614, 2016.

[12] R. Mostafa and A. M. Reza, "Application of dispersion entropy to status characterization of rotary machines," Journal of Sound and Vibration, vol. 438, no. 6, pp. 291-308, 2018.

[13] Y. Zhang, S. Tong, F. Cong, and J. Xu, "Research of feature extraction method based on sparse reconstruction and multiscale dispersion entropy," Applied Sciences, vol. 8, no. 6, pp. 888-910, 2018.

[14] S. Luo, W. Yang, and Y. Luo, "Fault diagnosis of a rolling bearing based on adaptive sparest narrow-band decomposition and RefinedComposite multiscale dispersion entropy," Entropy, vol. 22, no. 4, p. 375, 2020.

[15] Y. Jiang, C.-K. Peng, and Y. Xu, "Hierarchical entropy analysis for biological signals," Journal of Computational and Applied Mathematics, vol. 236, no. 5, pp. 728-742, 2011.

[16] K. Zhu and H. Li, "A rolling element bearing fault diagnosis approach based on hierarchical fuzzy entropy and support vector machine," Proceedings of the Institution of Mechanical Engineers, Part C: Journal of Mechanical Engineering Science, vol. 230, no. 13, pp. 2314-2322, 2016.

[17] R.-V. Sánchez, P. Lucero, R. E. Vásquez, M. Cerrada, J.-C. Macancela, and D. Cabrera, "Feature ranking for multifault diagnosis of rotating machinery by using random forest and KNN," Journal of Intelligent \& Fuzzy Systems, vol. 34, no. 6, pp. 3463-3473, 2018.

[18] M. Costa, A. L. Goldberger, and C. K. Peng, "Multiscale entropy analysis of complex physiologic time series," Physical Review Letters, vol. 89, no. 6, 2002.

[19] H. Azami, M. Rostaghi, D. Abasolo et al., "Refined composite multi-scale dispersion entropy and its application to biomedical signals," IEEE Transactions on Biomedical Engineering, vol. 64, no. 12, pp. 2872-2879, 2017. 
[20] Y. B. Li, M. Q. Xu, H. Y. Zhao et al., "Research on bearing diagnosis method based on hierarchical fuzzy entropy and improved support vector machine," Journal of Vibration Engineering, vol. 89, no. 1, pp. 184-192, 2016.

[21] https://csegroups.case.edu/bearingdatacenter/pages/ download-data-file.

[22] Y. B. Li, L. Wang, and L. Jiang, "Fault diagnosis of rolling bearing based on improved depth confidence network of PSO," Vibration and Shock, vol. 39, no. 5, pp. 89-96, 2020.

[23] X. Yan and M. Jia, "Intelligent fault diagnosis of rotating machinery using improved multiscale dispersion entropy and mRMR feature selection," Knowledge-Based Systems, vol. 163, no. 1, pp. 450-471, 2019. 